\title{
Cárie dentária e gênero em adolescentes
}

\section{Dental caries and gender in adolescents}

\author{
Danielle Medeiros Veiga Bonotto* \\ Tatiana Pegoretti Pintarelli** \\ Gabriela Santin ${ }^{* * *}$ \\ Gisele Ristow Montes ${ }^{*}$ \\ Fernanda Morais Ferreira ${ }^{* * * *}$ \\ Fabian Calixto Fraiz ${ }^{* * * *}$
}

\section{Resumo}

Objetivo: avaliar a influência do gênero na prevalência de cárie dentária, hábitos de higiene bucal, consumo e preferência ao açúcar no início da adolescência. Sujeitos e método: Foi desenvolvido um estudo transversal representativo de adolescentes (doze anos) no município de Araucária,PR. Para a avaliação da prevalência de cárie não tratada e da higiene bucal, foi utilizado o índice CPO-D (Dentes cariados perdidos e obturados) e o índice IHO-S (Índice de higiene oral simplificado), respectivamente. O questionário de frequência alimentar (QFA) foi utilizado para mensurar o consumo de açúcar, e uma versão modificada do Sweet Preference Inventory verificou a preferência paladar ao doce. Resultados: os meninos apresentaram uma maior presença de placa $(p<0,001)$ e menor frequência diária de escovação quando comparados com as meninas $(p=0,017)$. A presença de placa dental foi associada à prevalência de dentes cariados não tratados tanto em meninos $(p=0,004)$ como em meninas $(p<0,001)$. Houve uma associação entre o maior número de contatos diários de alimentos com sacarose e a maior prevalência de dentes cariados não tratados apenas nas meninas. Quanto ao consumo de alimentos com sacarose, preferência paladar pelo doce e experiência de cárie dentária, não foram encontradas diferenças significantes entre os gêneros. Conclusão: o gênero não interferiu na prevalência de cárie não tratada em adolescentes de doze anos de idade. A higiene bucal na adolescência foi influenciada pelo gênero. O consumo de alimentos com açúcar foi associado à prevalência de cárie não tratada apenas nas meninas.

Palavras-chave: Cárie dentária. Adolescente. Gênero e saúde. Placa dentária.

\section{Introdução}

Nas últimas décadas, houve um declínio nos índices de cárie dentária no mundo, mas ela ainda é a doença bucal mais comum e a maior causa de perda de dentes em adolescentes ${ }^{1}$. A cárie dentária apresenta etiologia multifatorial, sendo influenciada por aspectos biológicos, comportamentais e socioeconô$\operatorname{micos}^{2}$. A adolescência é um período dinâmico e de transições complexas ${ }^{3}$ em que os comportamentos relacionados à saúde se consolidam, entre eles, destaca-se a higiene bucal e os hábitos alimentares ${ }^{4}$.

Segundo uma revisão sistemática recente, ainda há uma lacuna na compreensão dos fatores que influenciam o comportamento em saúde bucal dos adolescentes $^{5}$. Alguns estudos demonstram que os hábitos de higiene bucal de adolescentes estão associados a fatores demográficos, sociais, comportamentais e psicológicos, tais como gênero, nível socioeconômico e autopercepção da saúde ${ }^{4}$.

Atualmente, tem crescido o interesse em entender como o gênero influencia aspectos da saúde geral e bucal, especificamente da cárie dentária ${ }^{6}$. O gênero pode mediar as atitudes dos adolescentes referentes a hábitos alimentares e higiene, podendo interferir na prevalência da cárie dentária. Estudos comprovam que meninas apresentam mais interesse na saúde bucal e que a percebem melhor do que meninos ${ }^{7}$.

No estudo da cárie dentária e fatores relacionados, compreender as questões associadas ao gênero permite o estabelecimento de medidas de orientação comportamental mais direcionadas e assertivas.

Mestranda em Odontologia, Departamento de Estomatologia, Universidade Federal do Paraná, Curitiba, Paraná, Brasil.

Mestre em Saúde Bucal da Criança e Adolescente, Departamento de Estomatologia, Universidade Federal do Paraná, Curitiba, Paraná, Brasil. Doutora em Odontologia, Departamento de Estomatologia, Universidade Federal do Paraná, Curitiba, Paraná, Brasil.

Doutora em Odontopediatria, Departamento de Odontopediatria e Ortodontia, Universidade Federal de Minas Gerais, Belo Horizonte. Minas Gerais, Brasil. ***** Doutor em Odontopediatria, Departamento de Estomatologia, Universidade Federal do Paraná, Curitiba, Paraná, Brasil. 
Este trabalho teve como objetivo investigar a prevalência de cárie dentária não tratada e de fatores relacionados, como hábitos de higiene bucal, consumo e preferência ao açúcar, no início da adolescência em função do gênero.

\section{Sujeitos e método}

\section{População e desenho de estudo}

Foi desenvolvido um estudo transversal representativo de escolares de 12 anos de idade, de ambos os gêneros, regularmente matriculados na rede de ensino de Araucária, no estado do Paraná, Brasil.

O cálculo amostral foi realizado por meio da fórmula de estimativa para proporção, utilizando-se a prevalência de 63,3\% para cárie dentária aos doze anos de idade $^{8}$, adotando-se um nível de confiança de $95 \%$, erro máximo aceitável de $5 \%$, e considerando-se a população total como sendo de 2072 escolares. Além disso, a amostra $(\mathrm{n}=304)$ foi acrescida em $60 \%$ para compensar o efeito cluster, obtendo-se como amostra mínima 487 crianças, e em $20 \%$ para compensar as possíveis perdas, resultando em 584 crianças.

Foi realizada uma amostragem aleatória por conglomerado em dois estágios: escolas e crianças. A amostra total foi distribuída nas oito regionais administrativas, na rede pública e privada, mantendo-se a proporção de alunos matriculados, visando assegurar a representatividade. Foram excluídos os estudantes que usavam aparelho ortodôntico fixo, apresentavam alguma condição debilitante no dia da coleta, recusaram-se a participar ou que apresentaram dados incompletos.

A fluoretação da água de abastecimento público ocorre no município desde o início da década de 1980, e a concentração média de flúor na área urbana é de $0,7 \mathrm{mg} / 1^{9}$. O presente trabalho foi aprovado pelo Comitê de Ética em Pesquisa da Universidade Federal do Paraná (n⿳0 740.075.09.06).

\section{Avaliação clínica}

Para a avaliação da prevalência de cárie dentária não tratada foi utilizado o componente c (cariado) do índice CPO-D, de acordo com os critérios de diagnóstico da Organização Mundial da Saúde ${ }^{10}$. Para avaliação de higiene bucal, utilizou-se o índice IHO-S (índice de higiene oral simplificado), de acordo com critérios de diagnóstico da Organização Mundial da Saúde.

Os exames clínicos foram realizados nas próprias escolas, por um único examinador previamente calibrado, sob luz natural, utilizando espelho bucal plano número 5 e a sonda padrão OMS-621, após, foi feita a secagem e a limpeza dos dentes com compressa de gaze estéril. Para a calibração, a análise interexaminador foi testada comparando-se o examinador com o padrão-ouro. Após quinze dias, o examinador repetiu o exame para cárie dentária (análise intraexaminador). Para o índice de higiene oral simplificado (IHO-S), só foi possível verificar a concordância interexaminador. Para a análise estatística foi utilizado o coeficiente Kappa, dente a dente.

\section{Avaliação de características comportamentais}

\section{Escovação e avaliação do consumo de alimentos com sacarose}

Os dados sobre frequência de escovação foram obtidos por meio de questões respondidas pelos adolescentes. O consumo de açúcar também foi avaliado pela aplicação de um questionário de frequência alimentar (QFA) ${ }^{11}$ aplicado aos adolescentes. Foram selecionados para compor o QFA os vinte alimentos mais citados, que continham sacarose na composição ou adicionada, durante a aplicação do inquérito recordatório de 24 horas ( $\mathrm{R} 24 \mathrm{~h}$ ) no estudo piloto $(\mathrm{n}=61)$, sendo o tempo de referência o último mês. Para a determinação do número de alimentos com sacarose de consumo diário, foram agrupados todos os alimentos, sólidos e líquidos, que tiveram a citação de consumo no QFA nas categorias "1 vez ao dia" ou "2 ou mais vezes ao dia" pelos adolescentes, com peso igual a 1 para as duas opções. O número de vezes que um mesmo alimento com sacarose foi consumido durante o dia pelo adolescente e o momento de contato não foram considerados.

\section{Avaliação da preferência paladar por doce}

A avaliação da preferência paladar ao doce foi realizada por meio de uma versão modificada do Sweet Preference Inventory ${ }^{12}$. Esse indicador avaliou a preferência com o uso de cinco diferentes concentrações (em Molar = M) de uma solução açucarada, que nesse caso foram modificadas, de acordo com Maciel et al. ${ }^{13}$ (2001), para 0,075 M, 0,15 M, 0,30 M, $0,6 \mathrm{M}$ e 0,9 M, por apresentarem maior capacidade discriminatória no estudo piloto. Foi acrescentada também uma opção sem açúcar $(0,0 \mathrm{M})$. O veículo utilizado para esse teste foi o chá da erva mate tostada e triturada (Mate Leão® 200g, Curitiba-PR, Brasil). Para a realização do teste, cada adolescente recebeu $30 \mathrm{ml}$ de chá, organizados em sequência crescente de concentração de açúcar. Os adolescentes consumiram bolacha água e sal entre a degustação das soluções para neutralizar o paladar e selecionaram a solução de chá de sua preferência ao final do teste. 


\section{Considerações éticas}

Este estudo foi aprovado pelo Comitê de Ética em Pesquisa da Universidade Federal do Paraná e desenvolvido de acordo com a declaração de Helsinki. Todos os responsáveis pelas crianças assinaram um termo de consentimento livre e esclarecido.

\section{Análises estatísticas}

Os dados coletados foram tabulados e organizados para serem tratados estatisticamente por meio do programa Statistical Package for the Social Sciences $^{\circledast}$ (versão 15.0; SPSS Inc., Chicago, IL, EUA).

Para verificar diferenças na prevalência de lesões de cárie dentárias não tratadas, nos contatos diários de alimentos com sacarose e na presença de placa visível em função do gênero, foram utilizados os testes Qui-Quadrado e Qui-Quadrado de tendência linear.

Para as análises estatísticas, as variáveis foram categorizadas como se segue: IHO-S "satisfatório" (IHO-S < 1) e "insatisfatório" (IHO-S $\geq 1$ ), presença de cárie dentária não tratada "sem" (nenhuma lesão de cárie dentária não tratada) e "com" (pelo menos uma lesão de cárie dentária não tratada), frequência de escovação dentária diária " $<2$ vezes ao dia" e " $\geq$ a 2 vezes ao dia", número de alimentos com sacarose consumidos diariamente de " 0 a 3"; "4 a 6 " $\mathrm{e}$ " $>6$ ", preferência paladar ao doce " Baixa" (0.0 e $0.075 \mathrm{~mol} / \mathrm{l})$; "Média" (0.15 e $0.30 \mathrm{~mol} / \mathrm{l})$; "Alta" (0.6 e 0.9 mol/l). O nível de significância adotado foi de $5 \%$.

\section{Resulltados}

O estudo obteve uma taxa de resposta de $92 \%$, totalizando 538 crianças, sendo 318 (59\%) meninas e $220(41 \%)$ meninos. As perdas foram principalmente em função da ausência da criança no dia do exame clínico. A calibração intra e interexaminadores para cárie dentária e interexaminador para IHOS obtiveram valores de kappa maiores do que 0,80 .

Existiu associação entre o gênero e a higiene bucal, sendo que os meninos apresentaram uma prevalência de placa dental (IHOS $\geq 1$ ) maior do que as meninas ( $p<0,001)$. Além disso, eles apresentaram uma maior prevalência de relato de frequência diária de escovação menor do que duas vezes ao dia quando comparados com as meninas $(\mathrm{p}=0,017)$ (Tabela 1). Quanto ao consumo de alimentos com sacarose, preferência paladar pelo doce e a experiência de cárie dentária não foram encontradas diferenças significantes entre meninos e meninas (Tabela 1).
Tabela 1 - Associação entre o gênero e as variáveis cárie dentária não tratada, higiene bucal, consumo de sacarose e preferência paladar ao doce, Araucária, Brasil $(n=538)$

\begin{tabular}{l|r|r|r}
\hline \multirow{2}{*}{ Variáveis } & $\begin{array}{c}\text { Masculino } \\
\mathrm{n}(\%)\end{array}$ & $\begin{array}{c}\text { Feminino } \\
\mathrm{n}(\%)\end{array}$ & $\begin{array}{c}\text { Valor } \\
\text { de } \mathrm{p}\end{array}$ \\
\cline { 2 - 4 } & \multicolumn{3}{c|}{ Dentes cariados não tratados } \\
\hline Sem & $114(51,8)$ & $180(56,6)$ & \multirow{2}{*}{$0,273^{*}$} \\
Com & $106(48,2)$ & $138(43,4)$ & \\
\hline \multicolumn{2}{l|}{ IHOS** } \\
\hline$<1$ & $88(40)$ & $186(58,5)$ & \multirow{2}{*}{$\mathbf{0 , 0 0 1 *}$} \\
\hline
\end{tabular}

\begin{tabular}{l|r|r|r}
\hline \multicolumn{4}{c|}{ Frequência diária de escovação } \\
\hline$>2$ & $139(63,2)$ & $168(52,8)$ & \multirow{2}{*}{$\mathbf{0 1 7}^{*}$} \\
$\leq 2$ & $81(36,8)$ & $150(47,2)$ & \\
\hline
\end{tabular}

Alimentos com sacarose com consumo diário

\begin{tabular}{l|r|r|r}
\hline 0 a 3 & $75(34,2)$ & $88(27,9)$ & \\
4 a 6 & $61(27,9)$ & $2,4)$ & $0,268 * * *$ \\
$>6$ & $83(37,9)$ & $125(39,7)$ & \\
\hline
\end{tabular}

Preferência paladar ao doce

\begin{tabular}{l|r|r|l}
\hline Baixa & $17(7,7)$ & $33(10,4)$ & \\
Média & $135(61,4)$ & $206(64,8)$ & $0,226 * * *$ \\
Alta & $68(30,9)$ & $79(24,8)$ & \\
\hline
\end{tabular}

* Teste Qui-Quadrado;** Índice de Higiene Oral simplificado;*** Teste Qui-Quadrado para tendência linear. Resultados significantes ao nível de 5\% marcados em negrito

Fonte: elaborado pelos autores com dados da pesquisa.

Os piores índices de placa estiveram associados à prevalência de dentes cariados não tratados tanto em meninos $(\mathrm{p}=0,004)$ como em meninas ( $\mathrm{p}$ $<0,001)$. Meninos e meninas com IHOS $\geq 1$ apresentaram, respectivamente, uma prevalência $54 \% \mathrm{e}$ $63 \%$ maior de dentes cariados não tratados do que aqueles com IHOS $<1$. Além disso, a prevalência de dentes cariados não tratados foi maior nas meninas (RP 4,17; IC 95\%: 2,50-6,95) e nos meninos (RP 6,35; IC 95\%: 4,02-10,04) que apresentavam uma frequência de escovação diária menor do que duas vezes ao dia (Tabela 2 ). 
Tabela 2 - Associação entre dentes cariados não tratados e as variáveis relacionadas com higiene dental, estratificada pelo gênero, Araucária, Brasil $(n=538)$

\begin{tabular}{|c|c|c|c|c|c|c|}
\hline \multirow{2}{*}{\multicolumn{2}{|c|}{ Variáveis }} & \multicolumn{3}{|c|}{ Cariados não tratados } & \multicolumn{2}{|c|}{ Análise univariada } \\
\hline & & $\begin{array}{l}\text { Não } \\
\mathrm{n}(\%)\end{array}$ & $\underset{n(\%)}{\operatorname{Sim}}$ & $\begin{array}{c}\text { Total }^{*} \\
\mathrm{n}(100 \%)\end{array}$ & $p^{* *}$ & $\begin{array}{c}\text { RP } \\
95 \%(I C)\end{array}$ \\
\hline \multicolumn{7}{|c|}{$\mathrm{IHOS}^{* * *}$} \\
\hline \multirow{2}{*}{ Masculino } & $<1$ & $56(64)$ & $32(36)$ & 88 & \multirow{2}{*}{0,007} & 1 \\
\hline & $\geq 1$ & $58(44)$ & $74(56)$ & 132 & & $1,54(1,13-2,11)$ \\
\hline \multirow{2}{*}{ Feminino } & $<1$ & $122(66)$ & $64(34)$ & 186 & \multirow{2}{*}{$<0,001$} & 1 \\
\hline & $\geq 1$ & $58(44)$ & $74(56)$ & 132 & & $1,63(1,27-2,09)$ \\
\hline \multirow{2}{*}{ Total } & $<1$ & $178(65)$ & $96(35)$ & 274 & \multirow{2}{*}{$<0,001$} & 1 \\
\hline & $\geq 1$ & $116(44)$ & $148(56)$ & 264 & & $1,60(1,32-1,94)$ \\
\hline \multicolumn{7}{|c|}{ Frequência de escovação diária } \\
\hline \multirow{2}{*}{ Masculino } & $>2$ & $68(84)$ & $13(16)$ & 81 & \multirow{2}{*}{$<0,001$} & 1 \\
\hline & $\leq 2$ & $46(33)$ & $93(67)$ & 139 & & $4,17(2,50-6,95)$ \\
\hline \multirow{2}{*}{ Feminino } & $>2$ & $133(89)$ & $17(11)$ & 150 & \multirow{2}{*}{$<0,001$} & 1 \\
\hline & $\leq 2$ & $47(28)$ & $121(72)$ & 168 & & $6,35(4,02-10,04)$ \\
\hline \multirow{2}{*}{ Total } & $>2$ & $201(87)$ & $30(13)$ & 231 & \multirow{2}{*}{$<0,001$} & 1 \\
\hline & $\leq 2$ & $93(30)$ & $214(70)$ & 307 & & $5,37(3,81-7,55)$ \\
\hline
\end{tabular}

*Frequências menores que 538 são devido a dados perdidos; ${ }^{\star *}$ Análise de regressão de Poisson univariada; ${ }^{\star \star \star}$ Índice de Higiene Oral simplificado; RP = Razão de prevalência; IC = Intervalo de confiança. Resultados significantes ao nível de $5 \%$ marcados em negrito

Fonte: elaborado pelos autores com dados da pesquisa.

Houve associação entre o maior número de contatos diários de alimentos com sacarose e a maior prevalência de dentes cariados não tratados quando considerado o grupo total. Quando analisado por gênero, essa associação somente foi significativa nas meninas (Tabela 3).

Tabela 3 - Associação entre dentes cariados não tratados e as variáveis consumo de sacarose e preferência paladar ao doce, estratificada por gênero, Araucária, Brasil $(n=538)$

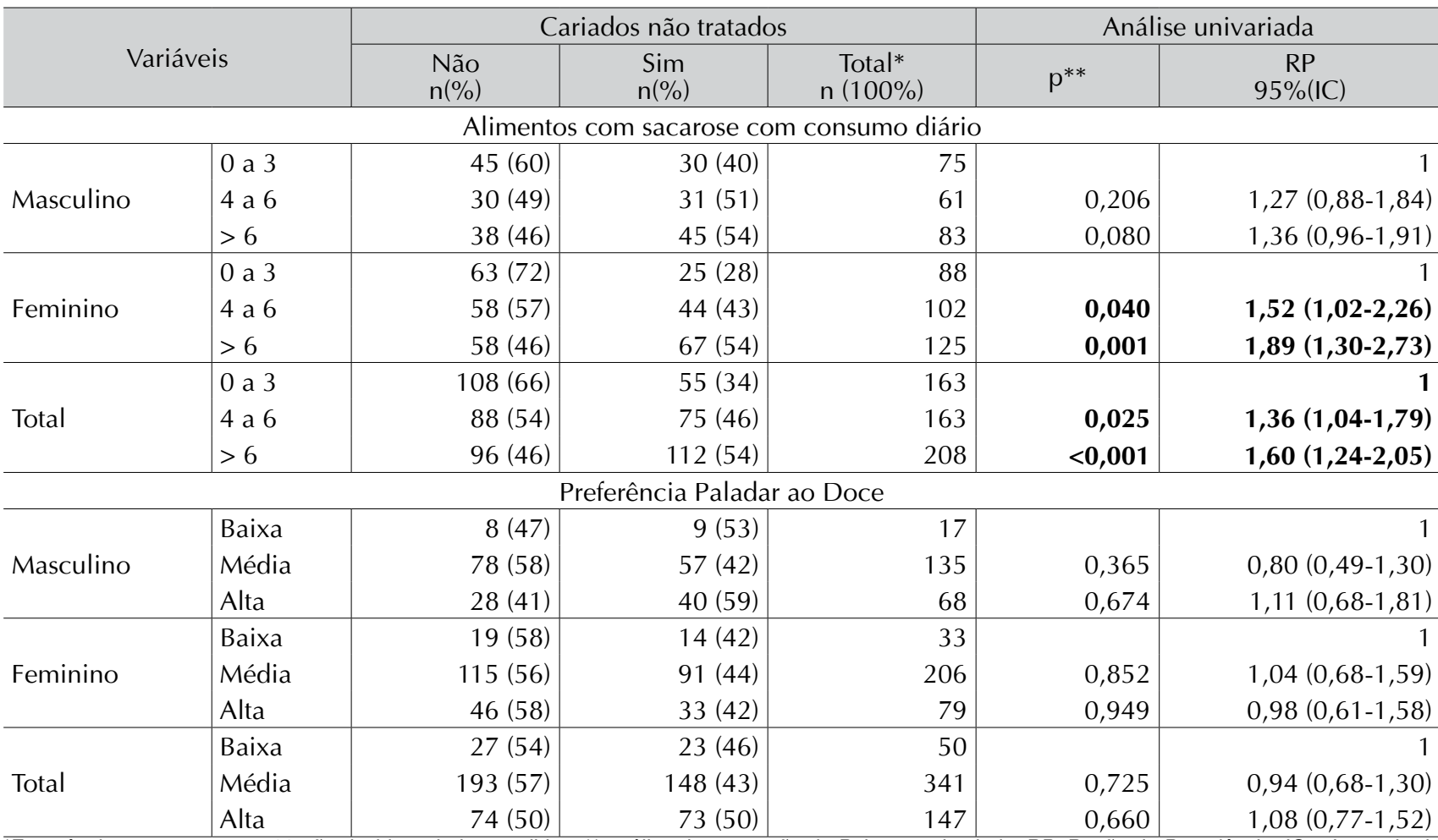

${ }^{\star}$ Frequências menores que 538 são devido a dados perdidos; ${ }^{\star \star}$ análise de regressão de Poisson univariada; RP=Razão de Prevalência; IC = Intervalo de confiança. Resultados significantes ao nível de 5\% marcados em negrito.

Fonte: elaborado pelos autores com dados da pesquisa.

\section{Discussão}

Nesse estudo, a prevalência da cárie dentária não tratada em meninos e meninas foi semelhante. Alguns trabalhos também não encontraram diferen- ça na prevalência de cárie em função do gênero ${ }^{14-16}$, enquanto outros, no entanto, observaram um maior acometimento da cárie no gênero feminino ${ }^{17,18}$, o que tem sido atribuído à composição e ao fluxo salivar, às flutuações hormonais, aos hábitos alimentares, às variações genéticas e a fatores culturais ${ }^{19}$. 
Um pior padrão de higiene bucal, analisado por meio de critérios clínicos e de autorrelato de frequência de escovação, foi associado a uma maior prevalência de cárie dentária em ambos os gêneros. A baixa motivação dos jovens de doze anos para a prática de hábitos bucais saudáveis pode ser reflexo da displicência no autocuidado, característica dessa fase ${ }^{20}$, o que torna essa população vulnerável ao acometimento da cárie, já que a presença de placa visível é um fator de risco importante para o desenvolvimento da cárie dentária ${ }^{21}$.

Neste estudo, as meninas apresentaram melhores indicadores de higiene bucal. Assim, é possível afirmar que o gênero exerce influência sobre os hábitos alimentares e de higiene bucal entre adolescentes $^{3,4,22}$, sendo que as meninas demonstram uma maior frequência da higiene bucal, uso do fio dental e de fluoretos ${ }^{3,6,20}$. Fatores socioculturais e psicossociais, além da maior preocupação feminina com aparência e limpeza, são apontados como responsáveis por essa associação $0^{4,5,19,22}$.

$\mathrm{Na}$ análise dos resultados deste trabalho, não houve associação entre preferência paladar e cárie dentária não tratada, mesmo quando estratificada por gênero. Embora a questão do paladar ou preferência por açúcares tenha sido considerada uma variável de valor explicativo para a ocorrência de cárie em crianças menores ${ }^{23}$, em adolescentes não foi detectada associação significante entre preferência paladar doce e prevalência de cárie ${ }^{13}$.

Também não foi constatada diferença significativa na frequência de consumo de sacarose entre os gêneros. A literatura não é conclusiva em relação à associação do gênero ao consumo de alimentos cariogênicos. Deve-se considerar que o comportamento alimentar de adolescentes é estruturado por influências individuais e ambientais, e o gênero é apenas uma das variáveis que mediam a decisão do que comer ${ }^{3}$.

Um estudo realizado com adolescentes paraibanos encontrou que as meninas, embora sejam mais conscientes sobre a relação entre dieta e cárie dentária, apresentam um maior consumo de doces ${ }^{24}$. Já entre adolescentes suecos, os resultados indicaram que o gênero não interferiu no consumo de alimentos com sacarose ${ }^{7}$. Entre os motivos para essas contradições provavelmente estão as características socioculturais e econômicas das populações estudadas, as quais podem interferir de forma decisiva no consumo de açúcar, minimizando as diferenças por gênero, especialmente nos primeiros anos da adolescência. No entanto, trabalhos com esse foco devem ser desenvolvidos para maior aprofundamento.

Como era esperado, houve associação entre o maior número de contatos diários de alimentos com sacarose e a maior prevalência de dentes cariados não tratados no grupo total. A frequência de consumo de alimentos com sacarose vem consistentemente sendo associada à experiência de cárie dentária em adolescentes de doze anos de idade ${ }^{25}$. É interessante observar que, quando estratificado por gênero, essa associação foi significativa apenas nas meninas, sendo que aquelas que apresentavam um maior consumo tinham uma prevalência de cárie maior. O padrão de erupção dentária mais precoce nas meninas ${ }^{11}$ pode ser um dos fatores explicativos desse aspecto, já que a manifestação da cárie é dependente do tempo de exposição aos fatores etiológicos.

Embora esse estudo, realizado em amostra representativa, tenha apresentado de forma clara as diferenças e semelhanças entre os gêneros, são necessários estudos longitudinais para avaliar se esses achados permanecem ou tendem a se modificar na medida em que os adolescentes caminham para a vida adulta. Outra limitação foi a avaliação de indivíduos no início da adolescência, em que o gênero ainda não apresenta uma grande influência. Além disso, deve-se salientar que parte dos dados foram obtidos por meio de autorrelato e, portanto, devem ser examinados com cautela.

O maior conhecimento em saúde pode promover mudanças de atitude e motivação para práticas e comportamentos saudáveis ${ }^{24,26}$, sendo a educação uma importante ferramenta para promoção e manutenção em saúde. O processo educativo deve ser direcionado para a especificidade da população que pretende atingir. Este trabalho colaborou para o entendimento de como o gênero interfere na prevalência de cárie, o que permitirá uma abordagem mais direcionada e efetiva.

\section{Conclusão}

O gênero não interferiu na prevalência de cárie não tratada em adolescentes de doze anos de idade. A presença de placa dental e o autorrelato de escovação foram associados à prevalência de cárie em ambos os gêneros. $\mathrm{O}$ consumo de alimentos com açúcar foi associado à prevalência de cárie não tratada apenas nas meninas.

A higiene bucal na adolescência foi influenciada pelo gênero, sendo que ações que visam melhorar o padrão de higiene bucal devem ter uma atenção especial para os meninos, reforçando conceitos de autocuidado.

\section{Abstract}

Objective: To assess the influence of gender on the prevalence of dental caries, oral hygiene habits, consumption and preference for sugar at the beginning of adolescence. Subjects and method: A representative cross-sectional study of adolescents (12 years old) was developed in the city of Araucaria, PR, Brazil. In order to assess the prevalence of untreated caries and oral hygiene, the DMFT and the OHI-S (Simplified Oral Hygiene) indexes were used, respectively. The Food Frequency Questionnaire (FFQ) was used to measure the consumption of sugar, and a modified version of the Sweet Preference Inventory was applied to investigate the preference for sweet flavors. Results: Boys presented 
higher presence of plaque $(p<0.001)$ and lower daily brushing frequency than girls $(p=0.017)$. The presence of dental plaque was associated with the prevalence of untreated decayed teeth in both boys $(p=0.004)$ and girls $(p<0.001)$. There was an association between the largest number of daily contacts with food with sucrose and the highest prevalence of untreated decayed teeth, only for girls. Regarding the consumption of food with sucrose, preference for sweet flavors, and dental caries experience, no significant differences were found between genders. Conclusion: Gender did not affect the prevalence of untreated caries in 12-year-old adolescents. Oral hygiene in adolescence was influenced by gender. The consumption of sugary food was associated with the prevalence of untreated caries, only for girls.

Keywords: Dental caries. Adolescent. Gender and health. Dental plaque.

\section{Referências}

1. World Health Organization. Oral Health. Fact sheet $\mathrm{N}^{\circ} 318$. Geneva: WHO; 2007. [citado 2015 mar. 15]. Disponível em URL: http://www.who.int/mediacentre/factsheets/fs318/en/.

2. Modéer T, Blomberg CC, Wondimu B, Julihn A, Marcus C. Association between obesity, flow rate of whole saliva, and dental caries in adolescents. Obesity (Silver Spring) 2010; 18(12):2367-73.

3. Story M, Neumark-Sztainer D, French S. Individual and Environmental Influences on Adolescent Eating Behaviors. J Am Diet Assoc 2002; 102(Suppl 3):40-51.

4. Freire MCM, Sheiham A, Bino YA. Hábitos de higiene bucal e fatores sociodemográficos em adolescentes. Rev Bras Epidemiol 2007; 10(4):606-14.

5. Calderon SJ, Mallory C. A systematic review of oral health behavior research in american adolescents. J Sch Nurs 2014; 30(6):396-403.

6. Doyal L, Naidoo S. Why dentists should take a greater interest in sex and gender. Br Dent J 2010; 209(7):335-7.

7. Östberg AL, Halling A, Lindblad U. Gender differences in knowledge, attitude, behavior and perceived oral health among adolescents. Acta Odontol Scand 1999; 57(4):231-6.

8. Brasil. Ministério da Saúde. Coordenação Nacional de Saúde Bucal. Projeto SB Brasil: condições de saúde bucal da população brasileira 2002-2003. Resultados principais. Brasília, 2004. 68p. [citado 2010 nov. 8]. Disponível em URL: <http:// bvsms.saude.gov.br/bvs/publicacoes/projeto_sb2004.pdf>.

9. Instituto Paranaense de Desenvolvimento Econômico e Social (IPARDES).Dados do município de Araucária 2006. [citado 2011 mar. 10]. Disponível em URL: <http://www.ipardes.gov.br/perfil_municipal/MontaPerfil.php?Municipio $=83700 \& b t O k=0 k>$.

10. Organização Mundial da Saúde. Levantamentos básicos em saúde bucal. 4. ed. Genebra; 1997. 66p.

11. Colucci ACA, Slater B, Philippi ST. Etapas para desenvolvimento de um questionário de frequência alimentar. Rev Bras Cien Saude 2005; 6:7-12.

12. Land DG, Shepherd R. Scaling and ranking methods. In: Piggott JR. Sensory analysis of foods. England: Elsevier; 1984. p. 141-77.

13. Maciel SM, Marcenes W, Sheiham A. The relationship between sweetness preference, levels of salivary mutans streptococci and caries experience in Brazilian pre-school children. Int J Paediatr Dent 2001; 11(2):123-30.
14. Willerhausen B, Blettner M, Kasaj A, Hohenfellner K. Association between body mass index and dental health in 1,290 children of elementary schools in a German city. Clin Oral Investig 2007; 11(3):195-200.

15. Alves LS, Susin C, Damé-Teixeira N, Maltz M. Overweight and obesity are not associated with dental caries among 12-year-old South Brazilian schoolchildren. Community Dent Oral Epidemiol 2013; 41(3):224-31.

16. Gerdin EW, Angbratt M, Aronsson K, Eriksson E, Johansson I. Dental caries and body mass index by socio-economic status in Swedish children. Community Dent Oral Epidemiol 2008; 36(5):459-65.

17. Chu $\mathrm{CH}$, Wang JH, Jan $\mathrm{RH}$, Huang $\mathrm{CH}$, Cheng CF. Association between health examination items and body mass index among school children in Hualien, Taiwan. BMC Public Health 2013; 19(13):1-7.

18. Antunes JLF, Junqueira SR, Frazão P, Bispo CM, Pegoretti T, Narvai PC. City-level gender differentials in the prevalence of dental caries and restorative dental treatment. Health Place 2003; 9(3):231-9.

19. Lukacs JR. Sex differences in dental caries experience: clinical evidence, complex etiology. Clin Oral Investig 2011; 15(5):649-56

20. Freddo SL, Aerts DRGC, Abegg C, Davoglio R, Vieira RC, Monteiro L. Hábitos de higiene bucal e utilização de serviços odontológicos em escolares de uma cidade da Região Sul do Brasil. Cad Saude Publica 2008; 24(9):1991-2000.

21. Rothen M, Cunha-Cruz J, Zhou L, Mancl L, Jones JS, Berg J. Oral hygiene behaviors and caries experience in Northwest precedent patients. Community Dent Oral Epidemiol 2014; 42(6):526-35

22. Ostberg AL, Halling A, Lindblad U. Gender differences in knowledge, attitude, behavior and perceived oral health among adolescents. Acta Odontol Scand 1999; 57(4):231-6.

23. Tomita NE, Nadanovsky P, Vieira ALF, Lopes E S. Preferências por alimentos doces e cárie dentária em pré-escolares. Rev Saude Publica 1999; 33(6):542-6.

24. Granville-Garcia AF,Fernandes LV, Farias TSS, D'avila S, Cavalcanti AL, Menezes VA. Adolescents' knowledge of oral health: a population-based study. Rev Odonto Cien 2010; 25(4):361-6.

25. Pereira SM, Tagliaferro EP, Pardi V, Cenci MS, Cortelazzi KL, Ambrosano GM, et al. Sugar consumption and dental health: Is there a correlation? Gen Dent 2010; 58(1):6-12

26. Brukiene V, Aleksejūniene J. An overview of oral health promotion in adolescents. Int J Paediatr Dent 2009; 19(3):163-71.

\section{Endereço para correspondência:}

Danielle Medeiros Veiga Bonotto

Rua Padre Anchieta, 2.454 sala 41 Bigorrilho 80730-000 Curitiba, PR

Telefone: 4130199256 / 99289982

E-mail: danidaniveiga@gmail.com

Recebido: 22/04/15. Aceito: 23/06/15. 